\title{
Commentaries
}

\section{A light at the end of the tunnel}

The article by van Laethem et al in this issue (see page 747) describes a well conducted study using the argon plasma coagulator (APC) to treat Barrett's oesophagus in 31 patients. However, complete eradication of Barrett's mucosa was only confirmed histologically in 19 (61\%), the remainder having a few residual Barrett's glands under the new squamous epithelium. Seventeen of those with apparent complete endoscopic and histological eradication at early follow up were biopsied again a year later, eight (47\%) of whom had relapsing islands of Barrett's metaplasia despite continuous treatment with omeprazole. These results lead to the same conclusion as that emerging from many recent studies on the endoscopic treatment of Barrett's oesophagus using a variety of techniques. ${ }^{1-5}$ It is possible to destroy specialised columnar epithelium in the oesophagus with a range of techniques and the treated areas heal with regeneration of squamous epithelium. However, there is a high risk of leaving some Barrett's glands under the regenerated squamous epithelium. This raises two crucial questions. Firstly, does it matter if a few glands are left behind and, secondly, can any of the treatment options be modified to give reliable destruction of all the Barrett's glands without any unacceptable complications.

The first question is tackled in the paper, although is very difficult to answer. Statistically, if the number of Barrett's glands is drastically reduced, one would expect the risk of neoplastic transformation also to be reduced, but this may be an over simplification. Columnar epithelium in the oesophagus could regenerate from the residual glands, and subsequently be vulnerable to neoplastic transformation. Only long term follow up of large numbers of patients such as those treated in this report can answer this question.

In the short term, there is more potential in looking at ways to improve treatment. The key is to find a technique that will destroy the full thickness of mucosa reliably without damaging the underlying muscle. In this paper, it is clear that there is no selectivity of effect between mucosa and muscle. This is not surprising as the biological effect of the APC is just due to local deposition of heat. The same would be expected with any technique that produces its effect by heating the tissue, such as multipolar electrocoagulation or a KTP laser. ${ }^{1}$ Two patients developed an oesophageal stricture severe enough to require dilatation, presumably because the APC had necrosed some muscle, which healed with scarring. The challenge is thus to deliver enough energy uniformly over the Barrett's oesophagus to destroy the mucosa but not to damage the muscle. As the narrow beam is moved over the area being treated under direct vision, and the result judged just on the immediate visual effect, I think it unlikely that it will be possible to improve much on the results described in this paper, especially as the thickness of the mucosa may vary with the degree of distension of the oesophagus at the moment each shot is fired.

A technique like photodynamic therapy (PDT) that treats all the mucosa uniformly would be more attractive. ${ }^{2-5}$ This involves systemic administration of a photosensitising drug and subsequent circumferential illumination of the affected segment of oesophagus with low power red light, usually from a laser. A variety of light delivery devices are being developed for this, including cylindrical transparent plastic devices and balloons with a central diffusing fibre that can be inflated in the oesophagus. ${ }^{2}$ The biological effect is photochemical rather than thermal and relatively sparing of connective tissues like collagen and elastin. However, studies using the photosensitising agent photofrin have shown that, as with the APC, there is no selectivity of necrosis between mucosa and muscle and strictures may occur. ${ }^{2}$

A more promising photosensitising agent for this application is 5-aminolaevulinic acid (ALA). In vivo, this is converted to the photoactive derivative, protoporphyrin IX (PPIX). In contrast to photofrin, which can be found in all layers of the oesophageal wall, PPIX localises mainly in the mucosa and experimental studies have shown that this can be exploited to give selective necrosis of mucosa without damage to underlying muscle. ${ }^{6}$ This is exactly what is required to treat circumferential zones of Barrett's oesophagus. Two important clinical studies have been published using ALA. ${ }^{34}$ Neither described any strictures after treatment, which suggests that the muscle layer is not affected, but both reported residual Barrett's glands under areas of regenerated squamous mucosa, so the problems are not yet all solved. Most clinical studies using ALA in the gastrointestinal tract have reported that the necrosis produced is very superficial using the maximum dose of ALA that can be tolerated by mouth $(60 \mathrm{mg} / \mathrm{kg}$, limited by first pass metabolism in the liver). ${ }^{7}$

Thus the next challenge is to find a way of increasing the depth of mucosal necrosis produced using ALA and seeing if this can be achieved without putting the muscle layer at risk. Options currently being explored are to give ALA intravenously, to increase the tissue concentration of PPIX either by using ALA esters ${ }^{8}$ or by adding an iron chelating agent (which slows down the final conversion of PPIX to haem), ${ }^{9}$ and to fractionate the light dose as experimental studies have shown that a single break of 150 seconds part way through illumination can increase the area of necrosis produced by a factor of four. ${ }^{10}$ However, none of these options has yet got beyond animal studies.

The results reported here by Van Laethem et al are the best that can be expected using the APC. If the few Barrett's glands remaining after treatment do not matter (and it will be years before we can answer this question), and occasional strictures are acceptable (as they seem to respond well to dilatation), the APC could become the treatment of choice as the procedure is relatively simple, safe and cheap. PDT with photofrin is probably inappropriate for Barrett's because of the risk of strictures and the long period of skin photosensitivity. It is more appropriate for treating small, locally invasive carcinomas. ${ }^{11}$ PDT with ALA has the greatest potential for complete destruction of Barrett's mucosa without causing strictures, but more basic work is required to find out how best to use it.

In the current state of knowledge, it is doubtful whether any form of endoscopic therapy for Barrett's oesophagus is appropriate other than in the context of clinical trials, unless there is evidence of severe dysplasia and the patient 
is considered a high risk for surgery. In the latter situation, endoscopic destruction of the mucosa by either thermal or photodynamic therapy can give worthwhile results.

National Medical Laser Centre, Department of Surgery, S G BOWN University College London Medical School, Charles Bell House, 67-73 Riding House Street, London W1P 7LD, UK

1 Barham CP, Jones RL, Biddlestone LR, et al. Photothermal laser ablation of Barrett's oesophagus: endoscopic and histological evidence of squamous Barrett's oesophagus: endoscopic and hist

2 Overholt BF, Panjehpour M. Photodynamic therapy for Barrett's oesophagus. Gastrointest Endosc Clin North Am 1997;7:202-20.

3 Barr H, Shepherd NA, Dix A, et al. Eradication of high-grade dysplasia in columnar-lined (Barrett's) oesophagus by photodynamic therapy with endogenously generated protoporphyrin IX. Lancet 1996;348:584-5.

4 Gossner L, Stolte M, Sroka R, et al. Photodynamic ablation of high-grade dysplasia and early cancer in Barrett's esophagus by means of 5-aminolevulinic acid. Gastroenterology 1998;114:448-55.
5 Laukka MA, Wang KK. Initial results using low-dose photodynamic therapy in the treatment of Barrett's esophagus. Gastrointest Endosc 1995;42.59-63. in the treatment of Barrett's esophagus. Gastrointest Endosc 1995; 42:59-63.
6 Loh CS, MacRobert AJ, Buonaccorsi G, et al. Mucosal ablation using photodynamic therapy for the treatment of dysplasia - an experimental study in the normal rat stomach. Gut 1996;38:71-8

7 Regula J, MacRobert AJ, Gorchein A, et al. Photosensitisation and photodynamic therapy of oesophageal, duodenal and colorectal tumours using 5 -aminolaevulinic acid induced protoporphyrin IX - a pilot study. Gut 1995;36:67-75.

8 Peng Q, Moan J, Warloe T, et al. Build-up of esterified aminolevulinic acid derivative induced porphyrin fluorescence in normal mouse skin. $\mathcal{F}$ Photochem Photobiol B 1996;34:95-6.

9 Curnow A, McIlroy BW, Postle-Hacon MJ, et al. Enhancement of 5-aminolaevulinic acid induced photodynamic therapy in normal rat colon 5 -aminolaevulinic acid induced photodynamic therapy in normal rat col
using hydroxypyridinone iron chelating agents. Br f Cancer (in press).

10 Messman H, Mlkvy P, Buonaccorsi G, et al. Enhancement of photodynamic therapy with 5 -aminolaevulinic acid induced porphyrin photosensitisation in normal rat colon by threshold and light fractionation studies. $\mathrm{Br} \mathcal{F} \mathrm{Can}$ cer 1995;72:589-94.

11 Sibille A, Lambert $\mathrm{R}$, Souquet $\mathrm{J}$, et al. Long term survival after photodynamic therapy for esophageal cancer. Gastroenterology 1995;108: 337-44.

\section{Progress in idiopathic bile acid malabsorption}

Bile acid malabsorption, whether caused by an inborn absence of the ileal transport system or by resection of the terminal ileum, causes diarrhoea because of the secretory effect of malabsorbed dihydroxy bile acids in the colon. There is now increasing evidence that bile acid malabsorption is also present and plays a causal role in many patients with intermittent or chronic diarrhoea who have no ileal pathology. Many of these patients have been labelled with the diagnosis of irritable bowel syndrome. ${ }^{1}$ In this issue, Fracchia et al (see page 812 ) provide new information on this syndrome by defining biliary bile acid and lipid composition in 13 well characterised patients with idiopathic bile acid malabsorption.

Bile acids are secreted by the liver entirely in conjugated form. The conjugated bile acid anion is impermeable to cell membranes and too large to pass the paracellular junctions of the biliary and intestinal tract. The highly efficient conservation of bile acids, which is responsible for the accumulation of a recycling bile acid pool, results from both carrier mediated and passive absorption. Carrier mediated absorption is mediated mainly by an apical transporter located in the ileal enterocyte. This transporter, which has been cloned and characterised by Dawson and colleagues, ${ }^{2}$ is now termed the "apical bile salt transporter" (abst) because it is also present in the renal tubular epithelial cell and in the cholangiocyte. Passive absorption of unconjugated bile acids that are formed by bacterial enzymes present in the distal small intestine and colon also contributes to the efficient conservation of bile acids. The combination of active and passive absorption of bile acids in the small intestine means that in health, probably less than $5 \%$ of bile acids that are secreted into the small intestine enter the colon.

The efficiency of bile acid absorption, the chemical complexity of the spectrum of conjugated and unconjugated bile acids that are absorbed, and the intrinsic variability of first pass hepatic extraction mean that detection of mild degrees of bile acid malabsorption by examining the spillover of bile acids into the systemic circulation is unlikely to be successful. What seems preferable is to measure what is not absorbed using a labelled bile acid that is absorbed solely by the ileal abst and is resistant to bacte- rial deconjugation. Ideally the label should have a short physical or biological half life and be easily measured.

All of these desirable attributes were achieved in a fruitful collaboration between George Boyd, Professor of Biochemistry at Edinburgh University, now deceased, and his radiochemical colleagues at Amersham who synthesised a derivative of cholic acid in which a ${ }^{75} \mathrm{Se}$ atom was inserted between the $\beta$ (C23) and $\gamma(\mathrm{C} 22)$ carbon atoms of the side chain. The resulting seleno-(homo)-cholic acid was conjugated with taurine to give seleno-homocholyltaurine, abbreviated SeHCAT. ${ }^{3}$ SeHCAT is handled by the liver and the intestine quite similarly to taurocholate (also called cholyltaurine), but is more resistant to bacterial deconjugation (and dehydroxylation).

How should this bile acid surrogate be used to detect ileal bile acid malabsorption? The most commonly used technique is to measure total body radioactivity before and seven days after administering SeHCAT. ${ }^{4}$ This approach is simple, but can be influenced by colonic transit time. ${ }^{56}$ Nonetheless, using ileal resection patients as the gold standard, Fellous and colleagues in a recent meticulous study $^{7}$ showed that the seven day SeHCAT retention test gave a sensitivity of $79 \%$ and a specificity of $90 \%$. Earlier, in an attempt to correct for variability in colonic transit, Ferraris et al administered SeHCAT together with a non-absorbable marker and determined the ratio in faeces. ${ }^{6}$ They showed that this approach was preferable because it was uninfluenced by colonic residence time.

A still more sensitive way of detecting bile acid malabsorption using SeHCAT is to measure the radioactivity located in the gall bladder area each day for several days. By plotting the natural logarithm of radioactivity against time, one can obtain the fractional turnover rate of the SeHCAT pool as the metabolism of most bile acids can be described by first order kinetics. Such an approach is based on the unproved assumption that most bile acids are stored in the gall bladder during the fasting state in the morning and that radioactivity in the gall bladder can be distinguished from that in the small and large intestine.

Fracchia and colleagues report measurements of biliary bile acid and biliary lipid composition in 13 patients with idiopathic bile acid malabsorption, and thus extend considerably our knowledge of this syndrome. The diagnosis of idiopathic bile acid malabsorption was based on the finding of an increased fractional turnover rate of SeHCAT and the lack of small intestinal pathology based on careful radiological and endoscopic studies including enteroclysis to exclude mucosal atrophy in the ileum. Fracchia et al found that biliary lipid composition, including biliary cholesterol saturation, and biliary bile acid composition did not differ significantly from those of control subjects who were 
studied similarly. Neither of these findings are so astonishing, but rational predictions should never substitute for experimental data. The enterohepatic circulation of bile acids should not be greatly perturbed in patients with idiopathic bile acid malabsorption, based on studies of patients with small ileal resections. Bile acid secretion is likely to decline modestly during the day; during overnight fasting, the increased hepatic synthesis should restore the bile acid pool to normal or near normal in size. Because the daily flux of bile acids through the hepatocyte is only modestly reduced, little change in biliary lipid secretion, the determinant of cholesterol saturation of bile, should occur. Patients with impaired transport function of abst can be expected to lose trihydroxy conjugated bile acids preferentially. However, the hepatocyte always synthesises trihydroxy bile acids preferentially, so that no great change in biliary bile acid composition should occur. Nonetheless, two of the patients did not have deoxycholic acid in bile. As deoxycholic acid is present in the biliary bile acids of most healthy adults, this finding suggests that the increased load of bile acids in the colon suppressed the growth of 7-dehydroxylating bacteria. A similar finding has been noted in some patients with bile acid malabsorption because of ileal resection.

Why should the busy gastroenterologist care about the presence of bile acid malabsorption in his patients with diarrhoea? Because treatment with a bile acid sequestrant may provide symptomatic benefit. In some patients the effective dose is quite small and dose titration is desirable. Some patients may prefer to ingest a sequestrant only when they are in a social situation where toilet facilities are remote. Some patients may prefer the inconvenience caused by increased faecal frequency and liquid stools to the unpalatableness of the resins. But all such patients should be relieved to know that their physician understands what is wrong and has a solution. New sequestrants are being developed that are considerably more potent than cholestyramine or colestipol. These will probably be available in capsule form and thus less distasteful than the currently available suspensions of resin granules. Relief of diarrhoea caused by bile acid malabsorption when a bile acid sequestrant was administered was described nearly 30 years ago, but the use of sequestrants for this purpose remains "off label" and has yet to be included in the colourful advertisements sponsored by the pharmaceutical industry.

As Hardison mentioned in an editorial over a decade ago, the availability of SeHCAT has helped to "illuminate a shadowy syndrome". ${ }^{8}$ It is less shadowy after this careful study of Fracchia et al. Progress in understanding why bile acid malabsorption is present in these patients will probably require sequencing of the abst gene in individual patients. Site directed mutagenesis studies in such transporters have shown that a change in a single base pair may cause a profound change in transport function. Multiple types of defects in gene product expression and targeting have been shown for the glucose-galactose transporter of the small intestine, and the same can be anticipated for abst. Probably, the powerful techniques of molecular biology will soon shed new insights into the defects present in patients with idiopathic bile acid malabsorption who were once thought to have little more than irritable bowels.

The author's work is supported by a grant from the National Institutes of Health (DK 21506) and a grant-in-aid from the Falk Foundation e.V., Freiburg, Germany

Division of Gastroenterology, Department of Medicine

A F HOFMANN

University of California, San Diego, USA

1 Galatola G and the Italian ${ }^{75}$ SeHCAT Multicentre Study Group. The prevalence of bile acid malabsorption in irritable bowel syndrome and the effect
of cholestyramine: an uncontrolled open multicentre study. Eur $\mathcal{F}$ Gastroenof cholestyramine: an uncont

2 Craddock AL, Love MW, Daniel RW, et al. Expression and transport properties of the human ileal and renal sodium-dependent bile acid transporter. Am ₹ Physiol 1998;37:G157-69.

3 Boyd GS, Merrick MV, Monks R, et al. Se-75-labeled bile acid analogs, new radiopharmaceuticals for investigating the enterohepatic circulation. $\mathcal{F} \mathrm{Nucl}$ Med 1981;22:720-5

4 Nylin H, Merrick MV, Eastwood MA, et al. Evaluation of ileal function using 23-selena-24-homotaurocholate, a $\gamma$ labeled conjugated bile acid. Initial clinical assessment. Gastroenterology 1983;84:63-8.

5 Hofmann AF, Bolder U. Detection of bile acid malabsorption by the SeHCAT test: principles, problems, and clinical utility. Gastroenterol Clin Biol 1994;18:847-51

6 Ferraris R, Jazrawi RP, Bridges C, et al. Use of a gamma labelled bile acid (75-SeHCAT) as a test of ileal function. Methods of improving accuracy. Gastroenterology 1986;90:1129-36.

7 Fellous K, Jian R, Hanniche M, et al. Mesure de l'absorption iléale des sel biliares par le test à l'homotaurocholate marqué au Sélenium 75 . Validation et signification clinique. Gastroenterol Clin Biol 1994;18:865-72.

8 Hardison WGM. Technology illuminates a shadowy syndrome. Gastroenterology 1986;91:242-4.

\section{Urokinase receptor is a key player in tumour progression}

Cell-cell and cell-extracellular matrix (ECM) interactions are essential for cell migration, tissue remodelling, angiogenesis, and tumorigenesis. Pericellular proteolysis of cell surface molecules and ECM provides crucial information in the local environment. Urokinase (uPA) plays an important role through the activation of plasminogen to plasmin, which regulates degradation of elements within the ECM, such as fibrin, fibronectin and lamin, and proteolytic activation of growth factors including hepatocyte growth factor (HGF), basic fibroblast growth factor (FGF-2) and transforming growth factor $\beta$ (TGF- $\beta$ ). Plasmin also activates the proenzyme forms of the matrix metalloproteinases (MMPs), such as MT1-MMP, ${ }^{1} \mathrm{MMP}-2$ and MMP-9. ${ }^{2}$ uPA activation is regulated by its specific cell surface receptor, urokinase receptor (uPAR). Binding of uPA to its receptor (uPAR) accelerates uPA activation from an inactive proenzyme (prouPA). The activity of plasminogen activators can be regulated by the specific inhibitors, plasminogen activator inhibitor 1 (PAI-1) and plasminogen activator inhibitor 2 (PAI-2). The urokinase system is implicated in tumour cell invasion on a basis of generally increased uPA activity in metastatic tumours. In particular, uPAR expression on the surface of neoplastic cells is crucial for tumour invasion and metastasis. Overexpression of UPAR has been reported in several types of human carcinoma including gastric cancer, pancreatic cancer and colorectal cancer, ${ }^{3-5}$ and a high UPAR concentration in resected colorectal tumours is an independent and significant prognostic factor for five year overall survival. ${ }^{6}$

During cell migration, the expression of the plasminogen activator system is upregulated. UPAR is distributed over the entire cell surface of non-migrating cells, whereas it is polarised towards the leading edge of migrating cells where it increases plasminogen activity to facilitate cell migration. Although UPAR is attached to the cell membrane only by a glycosyl phosphatidyl inositol (GPI) anchor, which is added during post-translational processing, it is also a genuine receptor which induces an intracellular signal. Recent studies have shown that binding of uPA to uPAR activates cellular protein tyrosine kinases, ${ }^{7}$ the protein kinase $\mathrm{C}$ pathway ${ }^{8}$ and mitogen-activated protein (MAP) 
kinases. ${ }^{9}$ Moreover, a direct signalling pathway utilising the Jak/Stat cascade and a second signal transduction mechanism via Src-like protein tyrosine kinases have been implicated in its signalling. ${ }^{10}$ Interactions of PAI-1 and UPAR with the extracellular matrix protein vitronectin (VN) and integrin receptors have been reported. Excess PAI-1 may promote cell migration by blocking cellular adhesion to $\mathrm{VN}$ and/or promoting detachment of uPARbearing cells, ${ }^{11}$ and uPAR changes the adhesive properties of integrins. UPAR forms stable complexes with activated integrins to inhibit their usual adhesive function and promote adhesion to VN mediated by the distinct binding site on UPAR. ${ }^{12}$ Thus, uPAR is a multifunctional cellular receptor that is involved in activation of proteolytic cascades, altered cell adhesion and intracellular signalling leading to tumour invasion and metastasis.

Various genetic abnormalities, including loss of function of tumour suppressor genes, activation of proto-oncogenes and deficiencies of DNA mismatch repair genes, are thought to contribute to steps in the adenoma-carcinoma sequence in colorectal neoplasia. Cellular transformation often results in a dramatic increase in the activation of the plasminogen activator system, especially urokinase. Kunz et al showed that wild type p53 represses transcription of the uPA and tPA gene through a non-DNA binding mechanism. ${ }^{13}$ However, the relation between uPAR expression and tumorigenesis has remained elusive. In this issue Suzuki et al (see page 798) show that uPAR expression increases during the transition from adenoma to invasive carcinoma in colorectal epithelium. uPAR expression was detected in $30 \%$ of colorectal adenomas and $85 \%$ of invasive carcinomas by in situ hybridisation. Importantly, they show that the frequency of uPAR gene expression was significantly increased in severely dysplastic adenomas compared with mildly or moderately dysplastic adenomas, and in Dukes' stage B or C cancer compared with Dukes' A cancer. These data implicate uPAR expression in the progression for normal colonic epithelium to an invasive carcinoma. How is the expression of this multifunctional cellular receptor regulated?

UPAR expression is upregulated by a variety of growth factors, including epidermal growth factor (EGF), FGF-2 and HGF, and by phorbol ester. Raised UPAR expression in colon cancer cells is largely a consequence of constitutive activation of the extracellular signal regulated kinases 1 (ERK-1) dependent pathway. ${ }^{14}$ Furthermore, our own data suggest that signalling through the tissue factor (TF)/factor VIIa (FVIIa) pathway results in upregulation of uPAR gene expression and enhanced invasion of pancreatic cancer cells in vitro. ${ }^{15}$ These data suggest that the expression of the components of the urokinase system depends upon a complicated series of paracrine interactions. Therefore, it is possible that uPA and/or PAI-1, which are expressed by local stromal cells, are assembled at the surface of the UPAR bearing tumour cells and the activated uPA system itself then contributes to paracrine regulation of growth factors and MMPs.

Clearly, the components of the urokinase system, especially uPAR, are attractive targets for cancer therapy.
Significant inhibition of cancer growth and neovascularisation by uPAR blockade has been observed for prostate cancer cell lines expressing mutant uPA. ${ }^{16}$ Reduction of uPAR on the cell surface, using an antisense strategy, has also been reported to induce a protracted state of dormancy in human epidermoid carcinoma cells. ${ }^{17}$ Thus, inhibition of the activated urokinase system may interfere not only with tumour invasion and metastasis but also with malignant progression. No doubt future studies will elucidate how these systems interact with each other, enabling the development of effective strategies for cancer therapy.

T TANIGUCHI N R LEMOINE

Imperial Cancer Research Fund Molecular Oncology Unit,

Imperial College School of Medicine,

Hammersmith Campus,

Du Cane Road, London W12 ONN, UK

A K KAKKAR

Department of Surgery,

Imperial College School of Medicine

Correspondence to: Professor Lemoine (email: n.lemoine@icrf.icnet.uk).

1 Okumura Y, Sato H, Seiki M, et al. Proteolytic activation of the precursor of membrane type 1 matrix metalloproteinase by human plasmin. A possible cell surface activator. FEBS Lett 1997;402:181-4.

2 Baramova EN, Bajou K, Remacle A, et al. Involvement of PA/plasmin system in the processing of pro-MMP-9 and in the second step of pro-MMP-2 activation. FEBS Lett 1997;405:157-62.

3 Park IK, Kim BJ, Goh YJ, et al. Co-expression of urokinase-type plasminogen activator and its receptor in human gastric-cancer cell lines correlates with their invasiveness and tumorigenicity. Int f Cancer 1997;71:867-73.

4 Cantero D, Friess H, Deflorin J, et al. Enhanced expression of urokinase plasminogen activator and its receptor in pancreatic carcinoma. Br f Cancer 1997;75:388-95.

5 Wang H, Skibber J, Juarez J, et al. Transcriptional activation of the urokinase receptor gene in invasive colon cancer. Int F Cancer 1994;58:650-7.

6 Ganesh S, Sier CF, Heerding MM, et al. Urokinase receptor and colorectal cancer survival. Lancet 1994;344:401-2.

7 Resnati M, Guttinger M, Valcamonica S, et al. Proteolytic cleavage of the urokinase receptor substitutes for the agonist-induced chemotactic effect. EMBO F 1996;15:1572-82.

8 Busso N, Masur SK, Lazega D, et al. Induction of cell migration by pro-urokinase binding to its receptor: possible mechanism for signal transpro-urokinase binding to its receptor: possible mechanism for
duction in human epithelial cells. $\mathcal{F}$ Cell Biol 1994;126:259-70.

9 Nguyen DH, Hussaini IM, Gonias SL. Binding of urokinase-type plasminogen activator to its receptor in MCF-7 cells activates extracellular signal-regulated kinase 1 and 2 which is required for increased cellular motility. F Biol Chem 1998;273:8502-7.

10 Dumler I, Weis A, Mayboroda OA, et al. The Jak/Stat pathway and urokinase receptor signaling in human aortic vascular smooth muscle cells. $\mathcal{F}$ Biol Chem 1998;273:315-21.

11 Waltz DA, Natkin LR, Fujita RM, et al. Plasmin and plasminogen activator inhibitor type 1 promote cellular motility by regulating the interaction between the urokinase receptor and vitronectin. F Clin Invest 1997;100:5867.

12 Wei Y, Lukashev M, Simon DI, et al. Regulation of integrin function by the urokinase receptor. Science 1996;273:1551-5.

13 Kunz C, Pebler S, Otte J, et al. Differential regulation of plasminogen activator and inhibitor gene transcription by the tumor suppressor p53. Nucleic Acids Res 1995;23:3710-17.

14 Lengyel E, Wang H, Gum R, et al. Elevated urokinase-type plasminogen activator receptor expression in a colon cancer cell line is due to a constitutively activated extracellular signal-regulated kinase-1-dependent signaling cascade. Oncogene 1997;14:2563-73.

15 Taniguchi T, Kakkar AK, Lemoine NR. Enhanced expression of urokinase receptor induced through the tissue factor-factor VIIa pathway in human pancreatic cancer. Cancer Res (in press).

16 Evans CP, Elfman F, Parangi S, et al. Inhibition of prostate cancer neovascularization and growth by urokinase-plasminogen activator receptor blockade. Cancer Res 1997;57:3594-9.

$17 \mathrm{Yu} \mathrm{W,} \mathrm{Kim} \mathrm{J,} \mathrm{Ossowski} \mathrm{L.} \mathrm{Reduction} \mathrm{in} \mathrm{surface} \mathrm{urokinase} \mathrm{receptor} \mathrm{forces}$ malignant cells into a protracted state of dormancy. $\mathcal{F}$ Cell Biol 1997;137:767-77.
Ever since it was generally concluded that endoscopic retrograde cholangiopancreatography (ERCP) and sphinc- terotomy was the treatment of choice for choledocholithiasis, big bile duct stones have remained a major challenge for the endoscopist. Extending a sphincterotomy increases risk of bleeding and perforation, mechanical lithotripters are generally expensive, cumbersome to use, fragile, and fail to grasp the stones effectively in a significant proportion of cases. Intraduct solvents such as mono octa- 
noin or methyl tert butyl ether are ineffectual or dangerous, or both. The somewhat defeatist approach of placing stents in elderly patients can lead to stent migration with occasional serious consequences or the formation of multiple stones above the stent, with the consequent risk of further cholangitis. There is probably a place at a few referral centres for extracorporeal shock wave lithotripsy (ESWL) but this requires some sort of biliary catheter to deliver $x$ ray contrast medium to facilitate targeting, which usually means at least two ERCPs in addition to the lithotripsy sessions. Furthermore, not all stones fragment easily and the capital costs and low usage mean that endoscopists must borrow sessions from their urological colleagues in most instances. One must not forget that surgery, despite its risks, may sometimes be the best option, but this is the ultimate admission of defeat by the endoscopist.

One approach which has spawned several publications but has not achieved much "market penetration" is contact lithotripsy. The initial, cheaper, electrohydraulic shock wave machines were rapidly shown to be very effective not only at fragmenting stones but also at punching holes through the bile duct wall unless every shot was clearly on target. ${ }^{12}$ Targeting is anything but easy when vision is obscured by clouds of gallstone fragments.

Subsequently a variety of laser techniques have been used. The coumadin pulsed-dye laser was intended, by choice of wavelength, to avoid damage to soft tissues through the elimination of absorption by haemoglobin but this was only partially successful and perforation can still occur, at least in animal models. ${ }^{3}$ Many ingenious mechanical devices, such as balloon catheters and wire guided Dormia baskets were developed in an attempt to improve targeting by $x$ ray monitors but in a three dimensional system such methods were always open to error and the use of miniscopes, delivered either percutaneously or via a "mother and baby" per oral system became more popular. Popular is only a relative term, however, for the difficulty of two skilled endoscopists, preferably close friends, manipulating one scope through the other in the deep recesses of the intrahepatic biliary tree while listening with a long stethoscope to check for good stone contact as one of them depresses the laser foot pedal while simultaneously observing both $x$ ray and endoscopy monitors and keeping the field of view constantly flushed of fragments, should not be underestimated. For these reasons the percutaneous approach gained ascendancy but both continue to suffer from the targeting problem which makes them very user unfriendly. ${ }^{45}$

The alternative approach, to develop a laser system which "knew" when it was aimed at a stone and only fired then was followed chiefly in Austria ${ }^{6}$ and Germany ${ }^{7} 8$ where at least three units have used the Rhodamine $6 \mathrm{G}$ pulsed dye laser with optical stone detection system produced by Telemit of Munich, including Hochberger et al who have published their latest results in this issue (see page 823). This technical advance means that it is no longer crucial to check that every shot of the laser is aimed correctly, as the power is extinguished after $5-8 \%$ of a shot is delivered, unless the returning reflected light indicates a hard target. Thus, radiologically assisted targeting, as in conventional ERCP, is all that is necessary and in their series of 60 patients accumulated over five and a half years, $87 \%$ fragmentation and duct clearance was achieved, albeit with five serious complications. In the three published series on this laser so far, 114 patients have been treated, 103 (90\%) successfully. The biggest news for endoscopists, however, is that 47 of the 60 patients in the present study were treated by conventional ERCP with $x$ ray fluoroscopic targeting alone. This blind firing does seem to increase the time required for fragmentation, however, as three times as many pulses are cut off on the grounds of bad targeting despite the use of balloons and wire guided baskets to improve aim. In their earlier study the authors admitted that one third of the patients required additional ESWL to achieve sufficient fragmentation for complete stone clearance. A corresponding figure is not available in the present study. Since sessions could incorporate up to one hour of laser firing and a mean of 1.8 sessions per patient, the procedure remains something of a test of endurance for both patient and staff.

However, according to Hochberger et al this paper is something of an historical document as they are currently using a piezo-acoustic stone detection system coupled to a Q switched NdYAG laser at only one third of the cost of the laser detailed in the present study and with greater effectiveness. ${ }^{9}$ Time will tell. In the meantime, why have so few centres purchased Rhodamine $6 \mathrm{G}$ lasers? I think at present that most people rely on the methods outlined in the first paragraph and until the "best buy" laser is built it would be better to wait a little longer. However, at least one or two centres in the UK should be investigating this additional method of managing large bile duct stones rather than leaving it all to our continental colleagues.

M L WILKINSON

Gastroenterology Unit,

5th Floor,

Thomas Guy House,

Guy's Hospital, London SE1 9RT, UK

1 Binmoeller KF, Bruckner M, Thonke F, et al. Treatment of difficult bile duct stones using mechanical, electrohydraulic and extracorporeal shock wave lithotripsy. Endoscopy 1993;25:201-6.

2 Bonnel DH, Liguory CE, Cornud FE, et al. Common bile duct and intrahepatic stones: results of transhepatic electrohydraulic lithotripsy in 50 patic stones: results of transhepatic
patients. Radiology 1991;180:345-8.

3 Nishioka NS, Kelsey PB, Kibbi AG, et al. Laser lithotripsy: animal studies of safety and efficacy. Lasers Surg Med 1998;8:357-62.

4 Cotton PB, Kozarek RA, Schapiro RH, et al. Endoscopic laser lithotripsy of large bile duct stones. Gastroenterology 1990;99:1128-33.

5 Ponchon T, Gagnon P, Valette PJ, et al. Pulsed dye laser lithotripsy of bile duct stones. Gastroenterology 1991;100:1730-6.

6 Schreiber F, Gurakuqi GC, Trauner M. Endoscopic intracorporeal laser lithotripsy of difficult common stones with a stone-recognition pulsed dye laser system. Gastrointest Endosc 1995;42:416-19.

7 Neuthaus H, Hoffmann W, Gottlieb K, et al. Endoscopic lithotripsy of bile duct stones using a new laser with automatic stone recognition. Gastrointest Endosc 1994;40:708-15.

8 Ell C, Hochberger J, May A, et al. Laser lithotripsy of difficult bile duct stones by means of a Rhodamine-6G laser and an integrated automatic stone-tissue detection system. Gastrointest Endosc 1993;39:755-62.

9 Hochberger J, Tschepe J, Tex S, et al. Basic investigations concerning a new low-cost piezo-acoustic stone-tissue-discrimination-system (paSTDS) for "smart" laser lithotripters and "blind" fragmentation of gallstones. Endoscopy 1996;28:S56. 